\title{
Network Centrality Measures and Systemic Risk: An Application to the Turkish Financial Crisis
}

\author{
Tolga Umut Kuzubas* \\ Boğaziçi University
}

\author{
Inci Omercikoglu ${ }^{\dagger}$ \\ Boğaziçi University
}

Burak Saltoglu
Boğaziçi University

December 18, 2013

\begin{abstract}
In this paper, we analyze the performance of several network centrality measures in detecting systemically important financial institutions (SIFI) using data from the Turkish Interbank market during the financial crisis in 2000. We employ various network investigation tools such as volume, transactions, links, connectivity and reciprocity to gain a clearer picture of the network topology of the interbank market. We study the main borrower role of Demirbank in the crash of the banking system with network centrality measures which are extensively used in the network theory. This ex-post analysis of the crisis shows that centrality measures perform well in identifying and monitoring systemically important financial institutions which provides useful insights for financial regulations.
\end{abstract}

Keywords: Systemic Risk, Network Centrality Measures, Systemically Important Financial Institutions, Turkey Financial Crisis in 2000

JEL Classification Numbers: F3, G1,D8

*Address: Boğaziçi University, Department of Economics, Natuk Birkan Building, 34342 Bebek, Istanbul, (Turkey). e-mail: umut.kuzubas@boun.edu.tr.

${ }^{\dagger}$ Address: Boğaziçi University, Department of Economics, Natuk Birkan Building, 34342 Bebek, Istanbul, (Turkey). e-mail: inci.omercikoglu@gmail.com.

¥Address: Boğaziçi University, Department of Economics, Natuk Birkan Building, 34342 Bebek, Istanbul, (Turkey). e-mail: burak.saltoglu@boun.edu.tr. 


\section{Introduction}

ln the last fifty years, we have experienced various global and local crises. The last financial crash in 2008 has confirmed the central role played by the interbank money markets for the smooth functioning of the financial system and implementation of monetary policy. Macro-prudential policies have led financial regulations to a new paradigm relying on a thorough investigation of the behaviour of the systemically important institutions(SIFI) through their role in affecting the entire system in the case of their failures.

Modern financial markets exhibit a high degree interdependence and interconnectedness. Those connections stem from agents balance sheets both for the asset and liability sides. However, the complex structure of the financial system makes it difficult to detect and monitor the institutions which are contributing to the systemic risk. In a comprehensive analysis of these interconnections for finance and insurance sectors, Billia et.al. (2010) proposed a formal measure which also captures linkages and balance sheet transactions. Besides, a recent study of G-20 shows that systemically important institutions are not only the largest ones but also those which are interconnected and capable of affecting the whole financial system ${ }^{1}$

There exist several econometric models to measure the systemic risk ${ }^{2}$. However, these tools and existing techniques at best give indirect indications of the system. Considering the fact that modern financial systems have a very complex structure, network representation gives the crucial picture of the whole system. Mapping financial systems to networks, taking financial institutions as nodes, network theory provides a promising framework for analyzing the inner working of interbank money flows.

A growing literature has emerged analyzing the stability of the interbank markets using network topological approach. This literature aims to identify institutions that are possibly systemically relevant because of the repercussions of their bankruptcy on other financial institutions. Therefore,

\footnotetext{
${ }^{1}$ See G20 London Summit, 2009, "Declaration on Strengthening the Financial System".

${ }^{2}$ See Bisias et. al. 2012
} 
it primarily investigates how different financial network structures respond to the default of a single financial institution to analyse which structures are more fragile and the characteristics of the institutions with a larger effect on the entire system in the case of their failures. HenggelerMuller (2006) argues that a financial institution, which is systemically important in a financial network, has the following characteristics: (i) Possesses many linkages to other members of the network (degree), (ii) The total amount of its assets, liabilities or flow in the network is very large (strength) (iii) Its failure could transmit contagion in a few steps (closeness), (iv) Its counterparts are considered also as relevant (eigenvector and PageRank) and (v) There are many paths which pass through it (betweenness). Therefore, network analysis is becoming an important tool for regulatory institutions and central banks to identify systemically important financial institutions, especially after the 2008 financial crisis. Applications stress the importance of network centrality measures in the "too interconnected too fail" context. In a recent paper, Leon and Perez (2013), assess the systemic importance for Columbian financial infrastructures by estimating authority and hub centrality. Bravo-Benitez et. al (2012), in order to monitor and measure systemic risk, investigates interbank payment system network in Mexico based on network centrality measures. Additionally, recent Basel III regulations puts a weight of $20 \%$ to interconnectedness of a bank in identification of Global Systemically Important Banks (G-SIB), hence interconnectedness and centrality is becoming an increasingly important tool for regulators to monitor systemic risk. ${ }^{3}$

Allen and Gale (2000) investigate the response of the banking system to a contagion when banks are connected under different network structures. Eisenberg and Noe (2001) analyze default by firms in an interbank market by introducing a single clearing mechanism. Gai and Kapadia (2007) develop a model of contagion using the network approach and assess the fragility of the financial system, depending on the degree of connectivity, and the liquidity of the market. Li et. al. (2010) introduces a network model and show that simulation of their model replicates features of real interbank networks such as low clustering coefficient and a relatively short average path length,

\footnotetext{
${ }^{3}$ see financial stability report by the Financial Stability Board, http://www.financialstabilityboard.org/publications/ $\mathrm{r}_{1} 31111$.
} 
community structures, and a two-power-law distribution of out-degree and in-degree. Soramaki et.al. (2007) investigates the network topological properties of Fedwire funds service and argues that network is scale-free and characterized by low average path length and low connectivity. Nier et.al. (2007) studies the relationship between network structure and number of defaults in a simulated random network model. In addition to this theoretical literature, an empirical literature studying the interbank networks has emerged which estimate bilateral credit relationships under different banking systems. Upper and Worms (2004) conducts an analysis for Germany, Cocco et.al. (2009) for Portugal, Degryse and Nguyen (2007) for Belgium, Wells (2004) for UK and Chang (2008) and Cajueiro and Tabak (2008) for Brazil. Basic premise of the literature is that shocks affecting central institutions are more likely to spread out to the entire system which suggests an intimate link between the position in the network and the contribution to the systemic risk. However, the main limitation of these papers is that they conduct counter-factual analysis based on simulations of the banking system and rely on the presupposition that more central institutions have a larger contribution to systemic risk, however this hypothesis is not tested in a real-life financial crisis. A notable exception along this dimension is Iyer and Peydro-Alcalde (2011) where they study the financial contagion due to interbank linkages using detailed balance sheet information from the failure of a large cooperative bank in India and show that interbank linkages act as an important channel of contagion.

In this paper, we also use a novel dataset from the Turkish banking crisis in 2000 which serves as a natural experiment to test the ability of network centrality in measuring systemic importance and contribution to systemic risk. The main contribution of our paper is measuring centrality of a financial institution, which is known as the main driving factor of the crisis, and introducing a time perspective where we follow this specific institution's centrality over time. We basically test the hypothesis whether existence of a central institution related to or associated with a financial crisis conditional on default of this institution.

During this crisis, the functioning of the interbank market changed drastically by a system- 
ically important institution (Demirbank) and which leads to a crash in the centralized network around this institution. ${ }^{4}$ The information on the timing of default of Demirbank which triggered the crisis through its default, allow us to compare properties of network measures retrospectively and facilitates the analysis of the performance network centrality measures in detecting and monitoring systemically important institutions. Using the same data set, Saltoglu and Yenilmez (2010) modifies the Google page rank algorithm and show that most interconnected financial institution can be detected five months before the crisis. We extend their analysis by introducing a broader set of centrality measures which are commonly used in the literature and assess their effectiveness. We also employ various network investigation tools such as volume, transactions, links, connectivity and reciprocity to gain a clearer picture of the network topology of the interbank market. Another contribution of our paper is to add a time-series dimension to the network analysis. By creating 240 consecutive networks based on daily transactions in the interbank market, we show that network centrality measures for Demirbank exhibits a significant increase in the period prior to the crisis hence perform well in identifying and monitoring systemically important financial institutions. Also, a recent approach introduced by Gencay and Signori (2012), Network Autoregression (NARMA), is a promising method to conduct conditional analysis based on data on the interconnections in the system to identify network effects. However, such an analysis requires detailed balance sheet information for the banks participating in the interbank network in our framework, thus due to data limitations we restrict our analysis with the centrality measures.

The remainder of the paper is organized as follows: Section 2 presents our data and descriptive statistics of the interbank market. Section 3 provides various network measures such as volume, transactions, links, connectivity and reciprocity applied to Turkish interbank market. Section 4 contains detailed investigation of the behaviour of centrality measures before and after the crisis. In section 5, we present a graphical analysis of the evolution of interbank network and section 6 concludes.

\footnotetext{
${ }^{4}$ See Danielsson and Saltoglu (2003), Gencay and Selcuk (2006) and Gradojevic and Gencay (2008) for an extensive analysis of the Turkish financial crisis in 2000.
} 


\section{Data and Descriptive Statistics}

In this section, we lay out the structure of the Turkish Interbank market and provide descriptive statistics. Recently, banks have started to lead higher loans and deposits daily due to globalization of the economies and amplified trade volumes worldwide. Higher loans and deposits are likely to cause imbalances in banks' balance sheets. Those imbalances can be compensated and corrected by short term, mostly overnight, interbank lending and borrowings. For example, a bank holding 3000 dollars in loans, 2200 dollars in deposits and 600 dollars in equity is likely to use interbank market to borrow additional 200 dollars to fund its balance sheet fully.

Topology of the interbank networks can be described as directed, weighted and built at a daily frequency. Generally, real money market networks are not strongly connected, i.e. each node is not reachable from every other node in the network. Furthermore, it is observed that not all links are reciprocal ${ }^{5}$. In our case, Turkish overnight money network between the years 2000 and 2001 exhibits a graph structure which is internally connected, directed but links are very rarely reciprocal.

Understanding the network structure of interbank market is important in determining banks' access, profitability and liquidity. It has been shown that interbank markets are not complete networks. In normal times, banks create relationships with other banks either through repeated transactions or through commitments to future lending. During crisis periods, banks check established relationships as a primary source of funding for additional liquidity. Prior and at the time of a crisis, interbank market faces many difficulties including liquidity shortages and credit quality fears. ${ }^{6}$ In the past, various G-20 Central banks have had to intervene to enable the banks continue accessing to fund.

We use data from Turkish overnight money market spanning the period 11 January 2000 and 21 December 2000 including interbank lending and borrowing volumes taken from the electronic

\footnotetext{
${ }^{5}$ For each loan transferred from bank A to bank B, another loan is also transferred back from B to A in the same day

${ }^{6}$ See Afonso et al., 2011 for an extensive discussion.
} 
interbank market of Istanbul Stock Exchange. There are 240 daily networks in which participating institutions are taken as nodes and their transactions are interpreted as linkages. There are 136 institutions participating in ISE overnight money market during the interval studied where 29 of those institutions in the network are excluded from the analysis because their total transaction volume fall behind 800000 dollars and constitutes a negligible fraction of total volume. Holidays are filtered due to abnormally less trading volume. Applying these filters, we end up with 107 institutions active in the interbank market.

Bid and ask prices along with the quantities are posted on the exchange. However, posting prices and quantities does not bind the agents i.e it is still acceptable to post a bid price and quantity to exchange and then not to sell it even though the expectations are met. Any trader can realize the transaction with any of the counter-parties with price and quantity posted on the common screen. In that sense, two agents bilaterally negotiate the trade which enables agents to limit lending and borrowing amounts to each specific party.

The data includes 1,355,604 tick by tick transactions of Turkish overnight money market. It is a package that has lended/borrowed amounts, strike prices and time of the transactions made. We simplify those 1,355,604 lines, first by clearing the transactions between different accounts of the same institution and then combining the transactions of same two agents. Besides, all transactions are stated in two duplicated lines differing only buyer/seller place. For example, to represent a transaction between agent DEM and YAT, there exist two lines in which DEM lies in a previous column and it is initialized as B (buyer) where YAT lies in latter column. This transaction can also be represented in a line in which YAT lies in previous column and initialized by letter S (seller) where DEM lies in latter column. Clearing these duplicated lines from data, we end up with a data set including 264,039 transactions for which date, time, volume, interest rates and identities of borrower and lender.

Descriptive statistics are provided in Table 2. We report average daily volume of total lending amounts and also include the proportion of lending made by the 25 largest market participants. 
Table 1: A Sample Transaction

\begin{tabular}{|l|c|c|c|c|c|r|}
\hline date & bank1 & seconds left & buyer/seller & bank2 & borrowed/lended amount & paid/gained amount \\
\hline 04.01 .2000 & DEM & 200001040000160 & B & YAT & 2000000000000 & 2001617830137 \\
\hline 04.01 .2000 & YAT & 200001040000160 & S & DEM & 2000000000000 & 2001617830137 \\
\hline
\end{tabular}

Based on the literature on the Turkish financial crisis, November 20 is taken as the first day of the financial breakdown. On November 20, based on our post-crisis knowledge, Demirbanks default created a gridlock in the borrowing channel through interbank markets and prevented borrowing of relatively healthier banks. Credit supply squeezed and demand amplified, and because of this asymmetry overnight rates increased to a level, which makes the market not operational. Therefore, we mainly focus on the behaviour of the market, before and after November 20, which we refer as pre and post-crisis periods.

The averages are about $20 \%$ in the pre-crisis period and $5-10 \%$ in the post crisis period. We observe that network evolves from a heavily centralized to a considerably less centralized on in the post-crisis period. Furthermore, starting from November 20, there is a significant decrease in total transaction volume and number of transactions.

Table 2: Monthly Summary Statistics

\begin{tabular}{|c|c|c|c|c|c|c|c|c|c|c|c|}
\hline & January & February & March & April & June & July & August & September & October & November & December \\
\hline Avarage Monthly Volume(mm TLs) & 1397925 & 1494891 & 1495500 & 1444724 & 1349803 & 1483989 & 1502739 & 1512345 & 1517382 & 1519374 & 13453921 \\
\hline Daily standard deviation of volume & 165559 & 175132 & 170835 & 168537 & 159441 & 158220 & 190837 & 193843 & 189374 & 237923 & 22039 \\
\hline Daily standard deviation of prices & 7,81 & 13,97 & 18,70 & 4,90 & 5,38 & 6,41 & 3,11 & 7,92 & 6,52 & 19,23 & 98,92 \\
\hline Number of loans & 55578 & 66740 & 60608 & 59220 & 69627 & 67783 & 65536 & 66273 & 64921 & 70183 & 71273 \\
\hline Largest 25 lenders in each month & & & & & & & & & & & \\
\hline Total lending(mm TLs) & 124689 & 118293 & 131479 & 128923 & 142783 & 153893 & 291831 & 300928 & 312393 & 279193 & 180383 \\
\hline Fraction of total & $21 \%$ & $20 \%$ & $19 \%$ & $22 \%$ & $21 \%$ & $21 \%$ & $23 \%$ & $21 \%$ & $24 \%$ & $25 \%$ & $22 \%$ \\
\hline
\end{tabular}

\section{Empirical Findings}

A detailed analysis of the structural changes between networks over time is hard to visualize. Therefore, in this section, we consider a set of statistical measures common in the network approach. Table 3 provides summary statistics of the interbank network. 
Table 3: Summary Statistics of Network Values

\begin{tabular}{lccccr}
\hline & Mean & Median & Max & Min & Std \\
\hline Volume & 2007,4 & 2093,7 & 3142,5 & 910 & 413,6 \\
Transactions & 824 & 773 & 1114 & 44 & 141,1 \\
\hline Nodes (buyer) & 23,5 & 25 & 34 & 13 & 4,40 \\
Nodes (seller) & 58,9 & 60,5 & 72 & 12 & 6,30 \\
Links & 286,4 & 288 & 374 & 30 & 42,43 \\
Connectivity & 4,76 & 4,80 & 6,22 & 0,49 & 0,70 \\
Reciprocity & 0,99 & 0,45 & 1,08 & 0,38 & 0,10 \\
\hline Link weight, vol. & 0,14 & 0,16 & 0,33 & 0,07 & 0,03 \\
Link weight, tran. & 0,35 & 0,68 & 0,38 & 0,22 & 0,06 \\
Node strength, vol. & 80,9 & 74,7 & 150,0 & 7 & 25,4 \\
Node strength, tran. & 42,5 & 42,2 & 56,8 & 2,25 & 7,11 \\
\hline
\end{tabular}

\subsection{Volume and Number of Transactions}

Volume represents the total amount of transactions made in each day in Turkish Liras where transaction figure represents the number of distinct transactions. The daily volume of the market can be considered as the sum of flows on directed links on a given day. Volume of transactions might be interpreted as a risk measure in the sense that high levels of transfer volume indicates that agents borrow and lend large amounts which makes them more connected and hence fragile to a contagion.

Figure 1 shows the volume of transactions in the Turkish overnight money market during 2000. We observe a significant increase in the market volume, with a peak on October 30, where Demirbank becomes the main borrower in the Interbank market. On November 20, there is drastic drop in the market volume because the level of overnight rate increased to a level which makes the market not operational. The bottom level is on December 5, when Demirbank is taken over.

We also plot transaction volume of Demirbank for the same time interval in Figure 2. It shows a similar pattern to the market volume which confirms that the volume increase during summer 


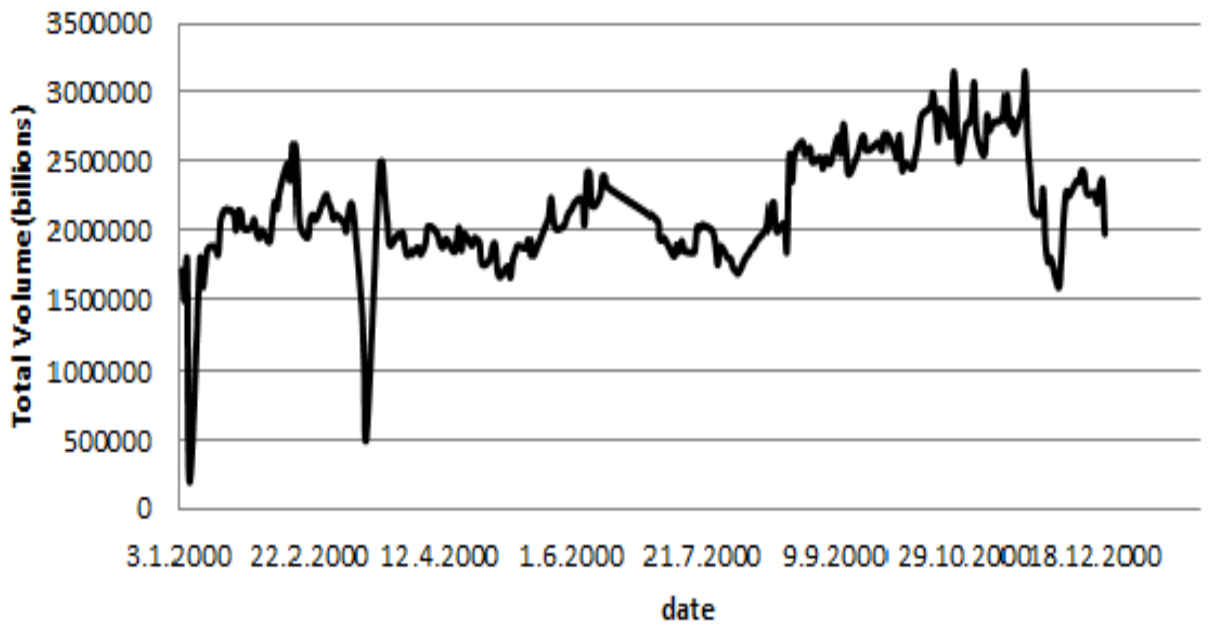

Figure 1: Daily volume of over night money market

months was due to Demirbank's start of its "bet" and the volume decrease in the post-crisis period was due to large decrease in Demirbank's borrowings.

We observe a similar pattern in the total number of transactions and the number of transactions by Demirbank in Figure 3 and 4, which confirms Demirbank's domination on the borrowing side of the market.

In order to obtain a clearer picture of the density of the network, we also use volume per transaction. Figure 5 shows that both total volume and number of transactions have increased in the pre-crisis period. We observe that in the pre-crisis period, increase in volume is not uniformly shared by participant institutions, hence there is an increase in volume per transaction which is normalized after the take-over of Demirbank.

\subsection{Links and Nodes}

An important characteristic of a node in a network is the number of links, which originate from a node and the number of links terminating in a node. In our analysis, we focus on the borrower side of the market and analysed the main borrower role of Demirbank to investigate the evolution of the interbank market. The time-series of the total number of links in the interbank network is 


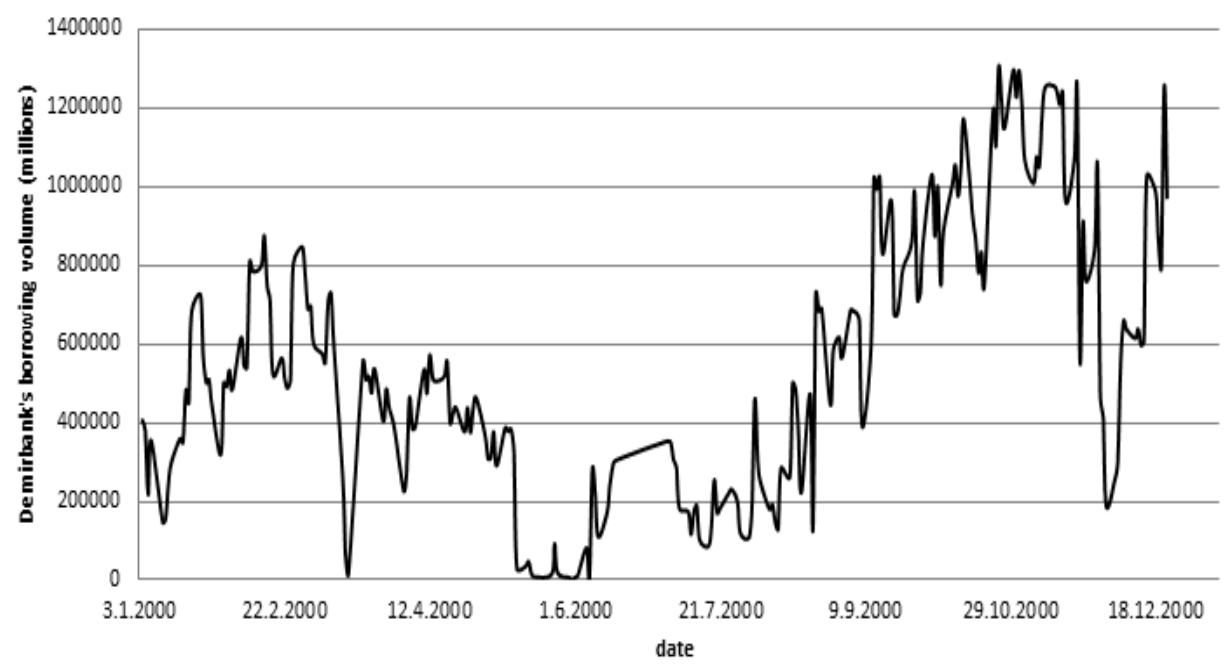

Figure 2: Daily borrowing volume of Demirbank

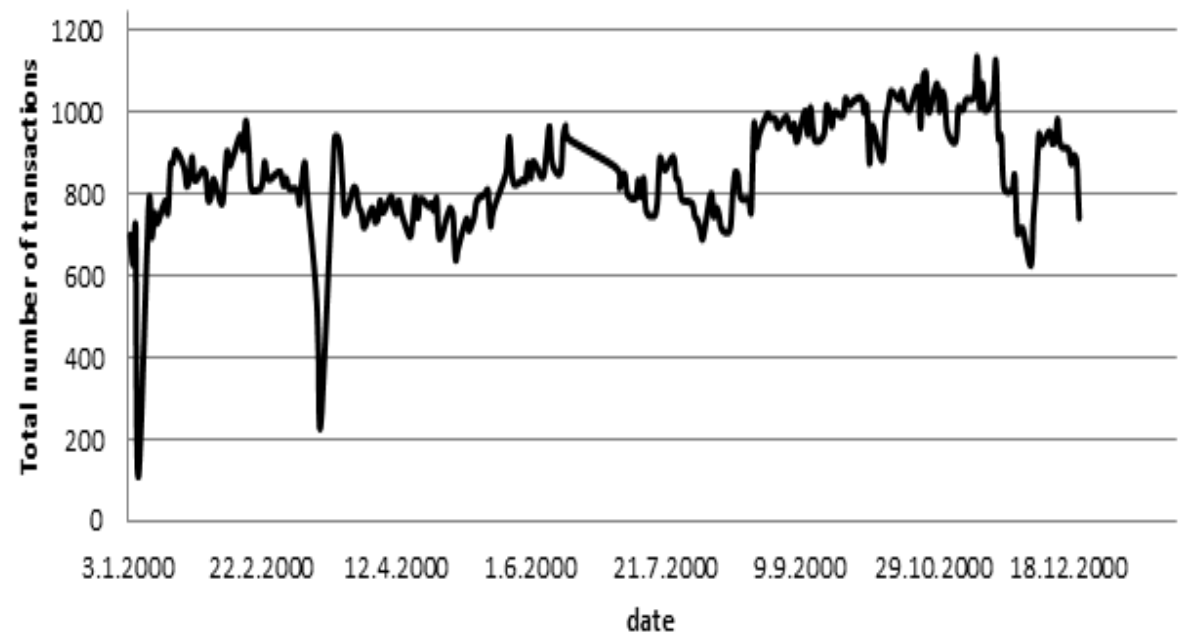

Figure 3: Total number of transactions 


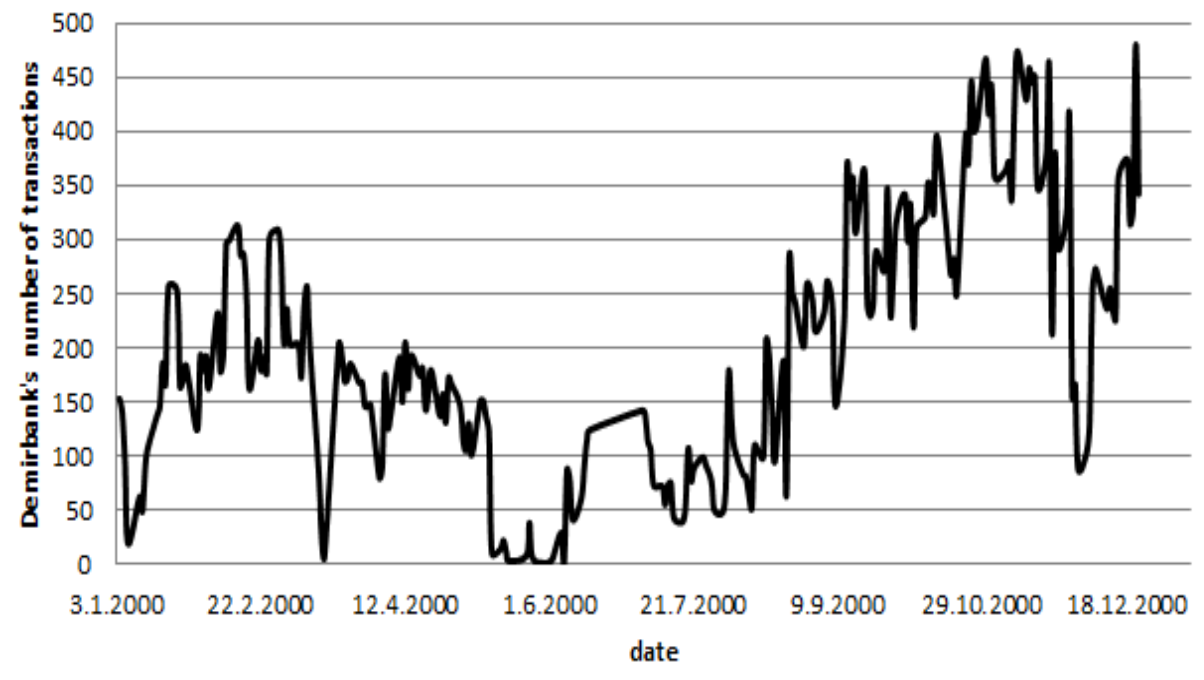

Figure 4: Demirbank's number of transactions

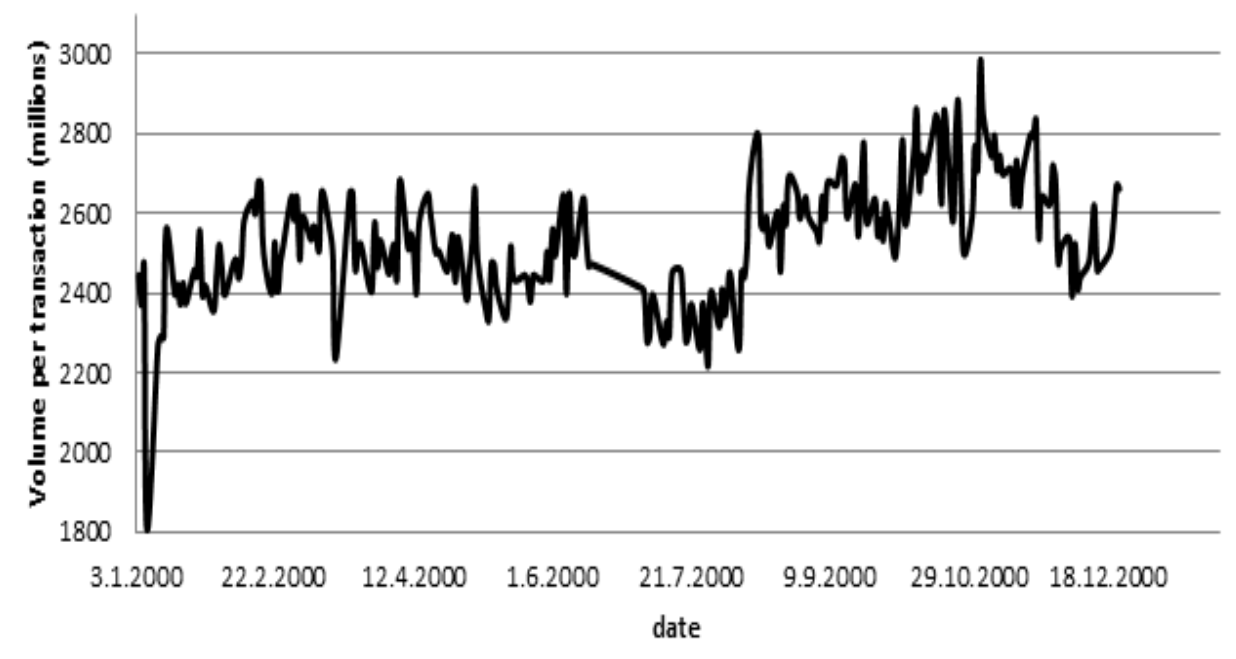

Figure 5: Volume per transaction 


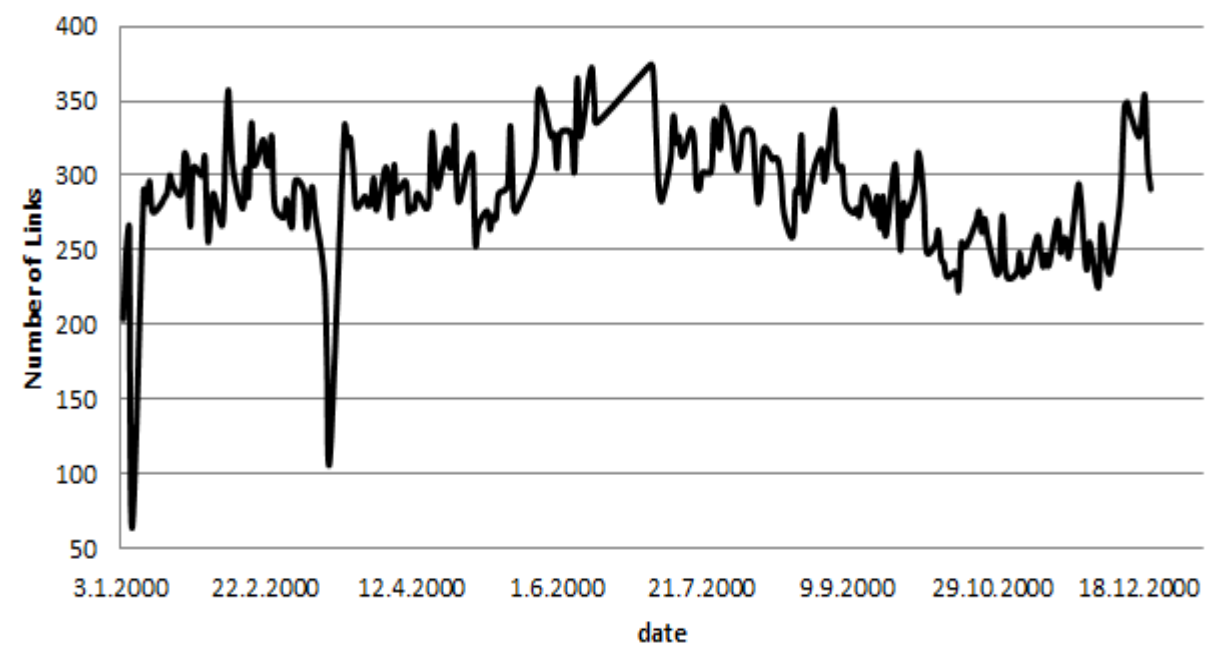

Figure 6: Total number of Links

given in Figure 6.

Number of links decreased in the pre-crisis period where the number of transactions increased. This pattern shows that trade is concentrated in larger volumes which contributes to the tension and risk at the market level.

In Figure 7, we plot the number of active borrowers. In the pre-crisis period, there is a significant decrease in the total number of borrowers in the interbank market, combined with the increase in Demirbank's number of transactions, confirms the domination of Demirbank on the borrowing side of the market.

\subsection{Connectivity}

We measure connectivity of the interbank network as the ratio of the total number of links to the number of potential links which is given as:

$$
C=\frac{E}{N(N-1)}
$$

where $C$ is the measure of graph connectivity, $N$ is the total number of nodes and $E$ is the 


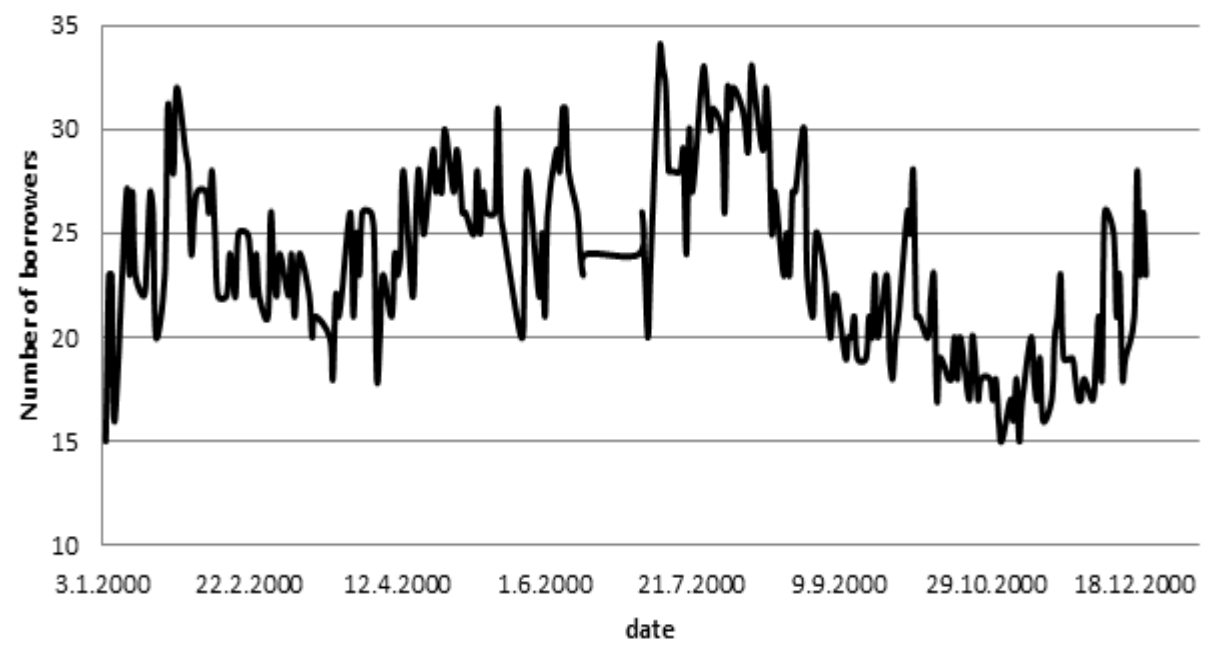

Figure 7: Total number of borrowers

total number of links.

Figure 8 provides a time-series representation of our connectivity measure. On October 30, we observe a peak in the connectivity of the network which corresponds to the time period where Demirbank becomes the main borrower in the interbank network. Combined with a significant drop in the total number of borrowers in Figure 7, interbank network becomes more connected while borrowing is concentrated among few banks. After October 30, as Demirbank becomes more risky to lend for the participants of the network, we observe a drop in the connectivity of the network.

Allen and Gale (2000) argues that connectivity is closely related to the stability of the interbank network and contagion. A more connected structure provides better risk-sharing opportunities for financial institutions, since in the case of a default of a particular bank, the effect of the negative shock is dispersed among other banks in the network. As observed in Figure 8, connectivity gradually declines towards November 20, which we identify as the first day of financial breakdown.

Another important measure is reciprocity which is the fraction of links for which the link with opposite direction exists in the network. Figure 9 shows the evolution of the reciprocity measure. We observe a lower average reciprocity in October and November, which confirms the 


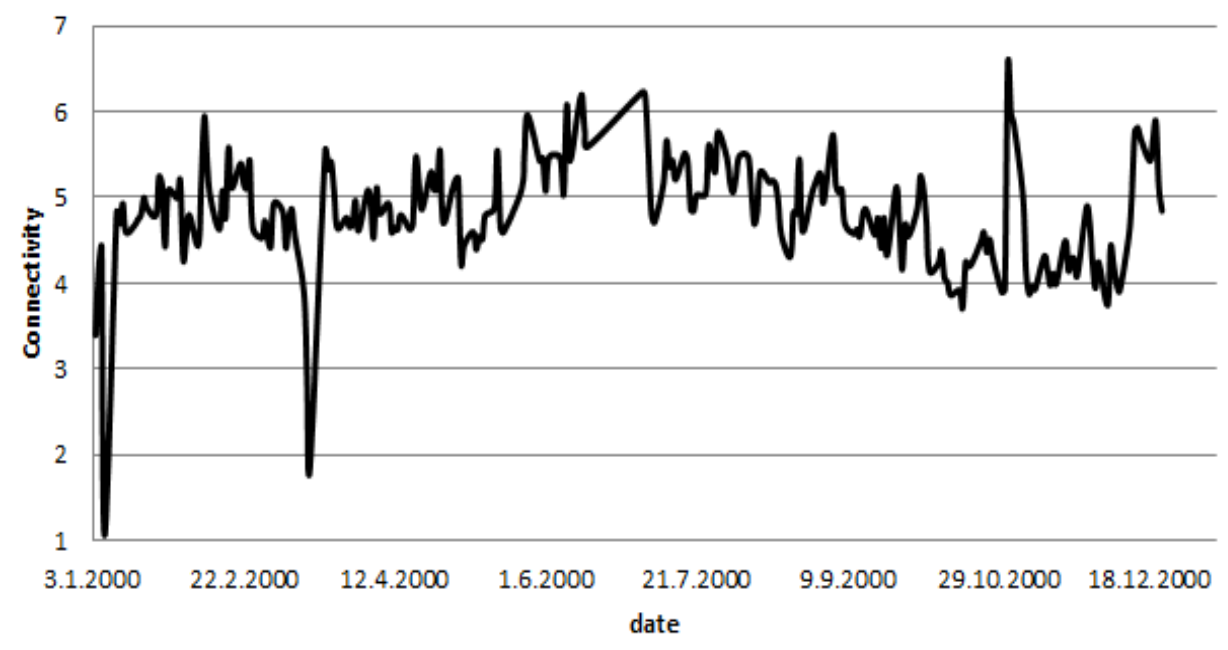

Figure 8: Connectivity

segmentation in the interbank network i.e. separated structure between lenders and borrowers. This segmentation contributes to the vulnerability of the system which becomes less flexible in absorbing shocks.

\section{Centrality Measures}

In this section, we empirically investigate the evolution of the centrality values for Demirbank, the critical institution which triggered the crisis, using four centrality measures, which are commonly used in network analysis: degree, betweenness, closeness, and Bonacich (eigenvector).

\subsection{Degree Centrality}

First and conceptually simplest centrality measure is the degree centrality which is defined as the number of links incident upon a node. The degree can be interpreted in terms of the immediate risk of a node for catching whatever is flowing through the network. We take in-degree counts as the degree measure because of our focus on the borrowing behaviour of Demirbank in the network. 


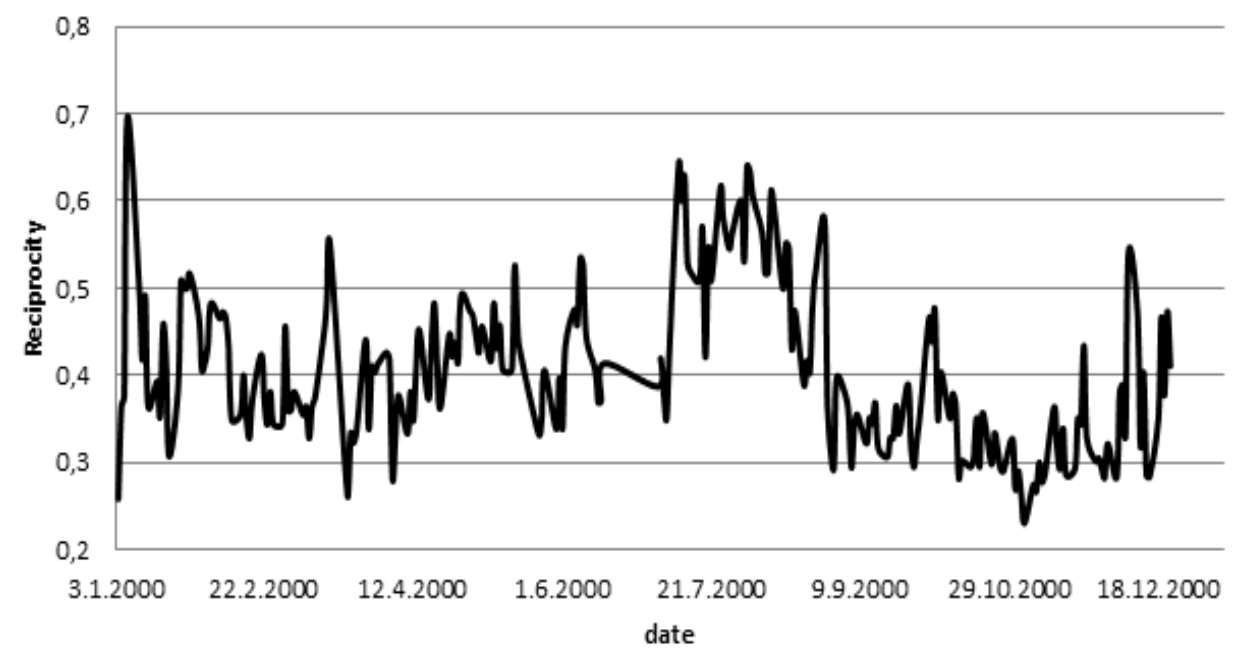

Figure 9: Reciprocity

From the beginning of July, Demirbank started to borrow from many different institutions which increases its degree centrality gradually reaching its peak on October 30 when Demirbank becomes the main borrower in the market. On December 1, when market volume drops to the bottom level for the time period we have studied, the degree centrality of Demirbank drops significantly due to precautionary credit squeeze by other institutions.

As in Figure 9, the total degree centrality of network, which is the number of formed links decreased until the take over. However, Demirbank's degree centrality increased during this period. This position continued until the take over on December 5 which leads to the normalization of the market.

\subsection{Closeness Centrality}

In this section, we investigate the closeness centrality values of Demirbank over time. We measure closeness centrality based on the distance of each bank to every other bank in the network. We compute closeness centrality for bank $i$ as: 


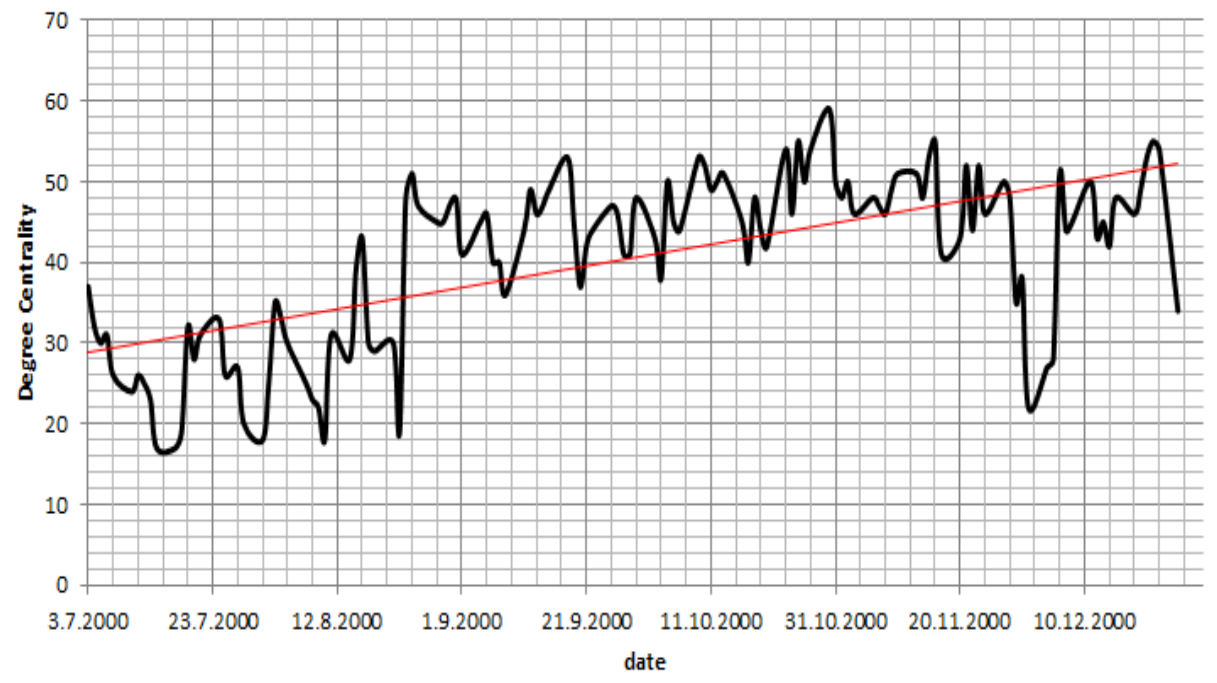

Figure 10: Degree centrality values of Demirbank

$$
C\left(b_{i}\right)=\left[\sum_{j=1}^{g} d\left(b_{i}, b_{j}\right)\right]^{-1}
$$

where $d$ is the path distance between banks $i$ and $j$.

An institution is considered important if it is relatively close to all other institutions. Closeness centrality indicates the influence of a node on the entire network. We observe that closeness centrality values of Demirbank have an increasing trend. Again the peak values are attained just before the crisis and they fell sharply afterwards.

The red line in Figure 11 shows the maximum closeness centrality value attained in the network whereas black line shows the closeness centrality value of Demirbank. It should be noted that as of the mid September, Demirbank leads the market i.e the maximum closeness values belong to Demirbank. This situation ends in the post-crisis period since the Demirbank is the most negatively affected institution. Thus, Demirbank's values are below the maximum values. Within 124 days, Demirbank has been the leader in terms closeness centrality in 67 days. 


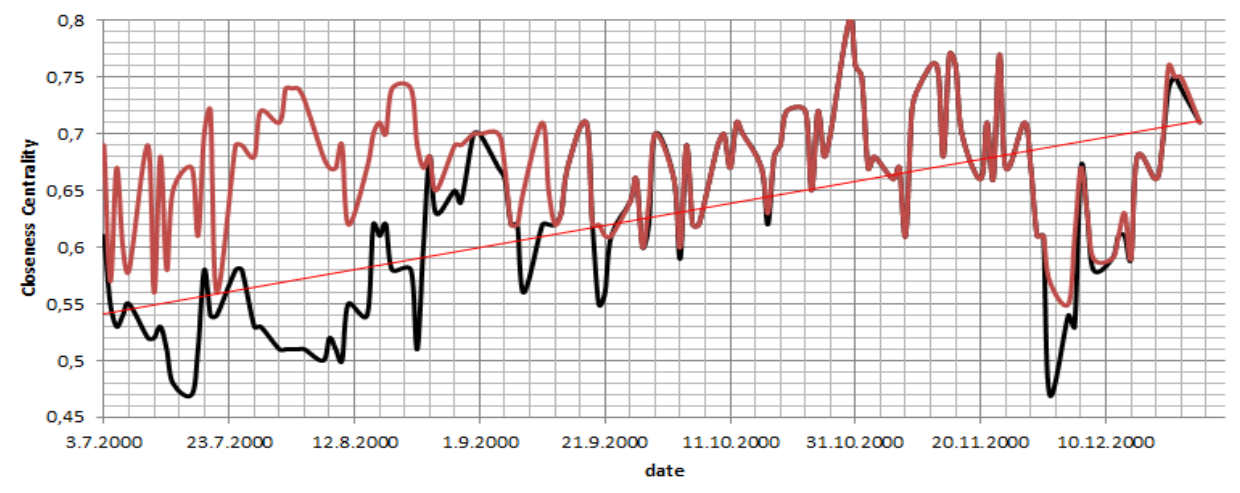

Figure 11: Closeness centrality values of Demirbank

\subsection{Betweenness Centrality}

Betweenness centrality of bank $i$ in the network is measured as:

$$
B\left(b_{i}\right)=\sum_{j<k} g_{j k}\left(b_{i}\right) / g_{j k}
$$

where $g_{j k}$ the number of shortest paths between $j$ and $k$ and $g_{j k}\left(b_{i}\right.$ the number of shortest paths between banks $j$ and $k$ that bank $i$ resides on.

Betweenness centrality builds on the notion that a vertex is central if it is needed to connect other pair of vertices. A node with high betweenness centrality can potentially influence the spread of information through the network. The betweenness centrality of Demirbank increases as it becomes the main borrower in the market. The value reaches the peak again on October 30 . During the crisis the centrality values decreased below the maximum value and increased after the normalization of the market on December 5. Within 124 days, Demirbank has been leader in terms of betweenness centrality in 68 days.

\subsection{Bonacich's Centrality}

We measure Bonacich centrality using the formula: 


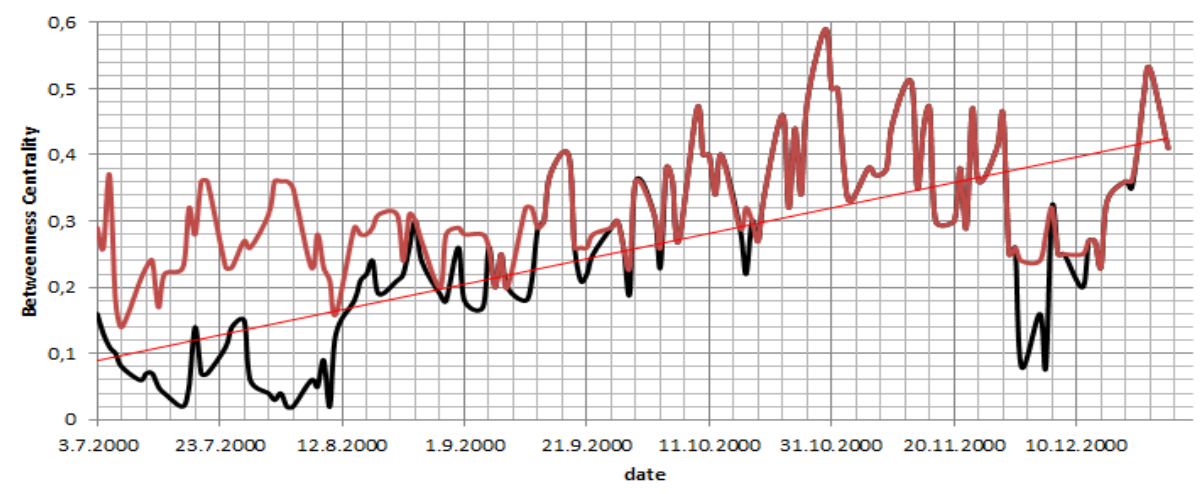

Figure 12: Betweenness centrality values of Demirbank

$$
C(G, \beta)=(I-\beta G)^{-1} G 1
$$

where $\beta$ is the weight, $G$ is the adjacency matrix and $I$ is the identity matrix.

Bonacich's centrality which is also known as the eigenvector centrality is based on the idea that a node is more central when there are more connections within its local network. ${ }^{7}$ More connections in its local area means that node is more powerful. This also means that power comes from being connected to those that are powerless. It assigns relative scores to all nodes in the network based on the principle that connections to high-scoring nodes contribute more to the score of the node in question than equal connections to low-scoring nodes.

Our focus in again on the centrality measures of Demirbank. For each network of transactions, we calculate Bonacich centrality values. Figure 13 plots the evolution of the Bonacich centrality values in the pre and post-crisis periods. We observe, a relatively milder and more volatile increasing trend which reaches its peak at October 30 similar to other centrality measures.

\footnotetext{
${ }^{7}$ See Bonacich (1972)
} 


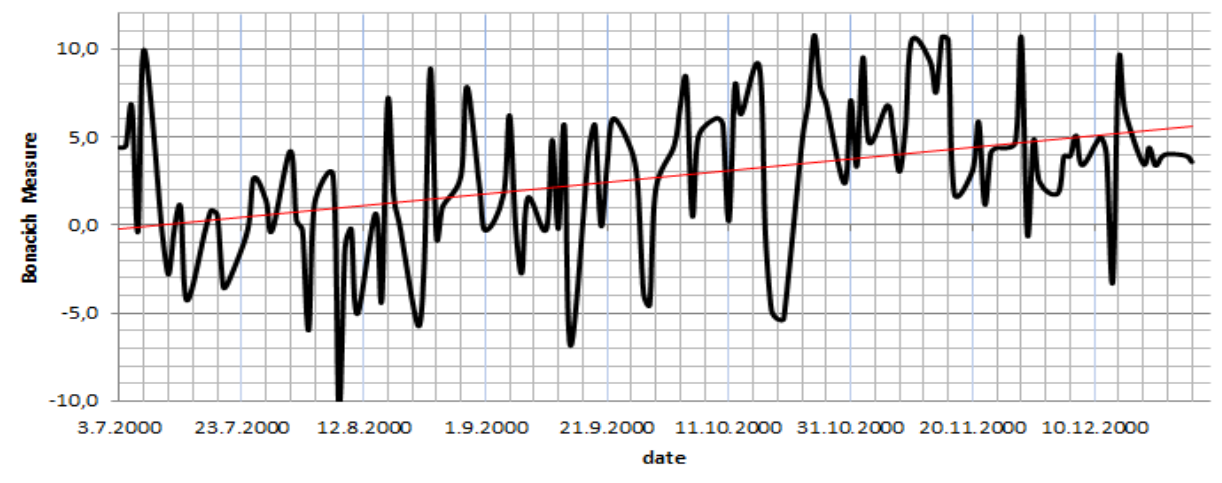

Figure 13: Bonacich centrality values of Demirbank

\section{Graph Representations}

In this section, we provide graph representations for selected days, before and after the crisis, to visualize the evolution of the interbank network. Based on the literature on the Turkish financial crisis in 2000, November 20 is identified as the first day of the financial downturn. In order to make the exposition clearer, we focus on banks making transactions over 100000 Turkish Liras and selected five days namely, February 28, October 30 , November 20, December 1 and December 5.

February 28 represents the time period where Demirbank is not the main borrower in the interbank market and the interbank network exhibits a multi-centered structure. ${ }^{8}$ There exists some central nodes, i.e. two borrowing centers. Since there are many other local centers in distinct neighbourhoods, those centers are not concentrated as hubs. Note that neither of these centers is Demirbank(DEM). However, Demirbank is one of the active banks in the network.

October 30 belongs to the period prior to November 20 and provides a clear picture of the Turkish interbank market before the crisis, the day where centralization became visible. Demirbank borrows from almost all of the participants of the interbank market and becomes the main borrower. It can be observed that almost all other banks in the network are lending to Demirbank

\footnotetext{
${ }^{8}$ Interbank market network is a scale free one, with a degree distribution which follows a power-law distribution with the scale parameter estimated as 2.97 .
} 
Figure 14: Circular layout representation of 28 February 2000

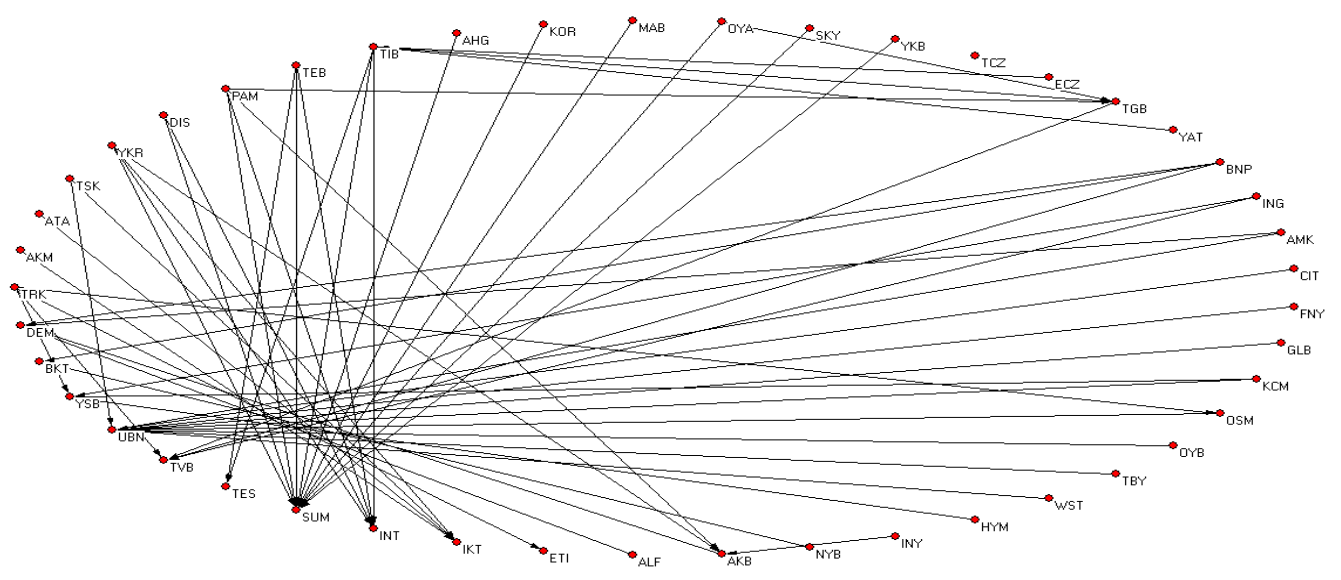

Figure 15: Circular layout representation of 30 October 2000

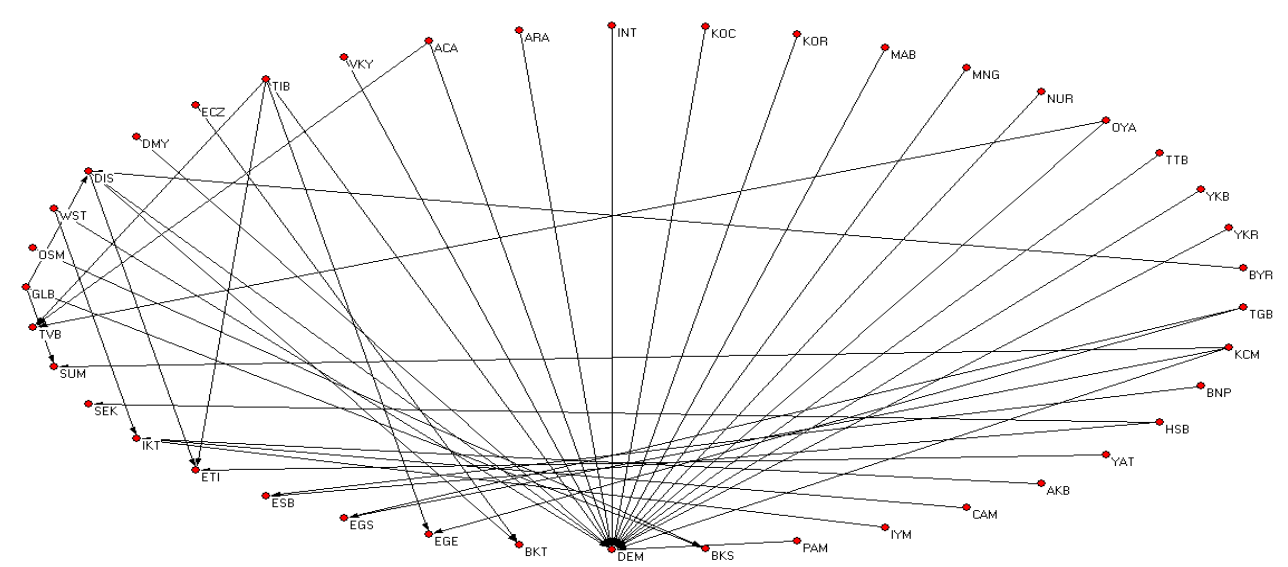

i.e. lending side of the market has a multi-agent structure.

November 20, which is the first day of the financial downturn, we observe that there are two centers in the network and the main borrower position of Demirbank is weakened. The closeness, betweenness and degree centrality values for Demirbank all decreased and fall behind of the values of other main borrowers.

On December 1, market volume drops to the bottom level for the time period we have studied, since banks refrain from making transactions. Demirbank becomes an isolated point in the network with connectivity, betweenness and degree measure values equal to zero, because banks stopped 
Figure 16: Circular layout representation of 20 November 2000

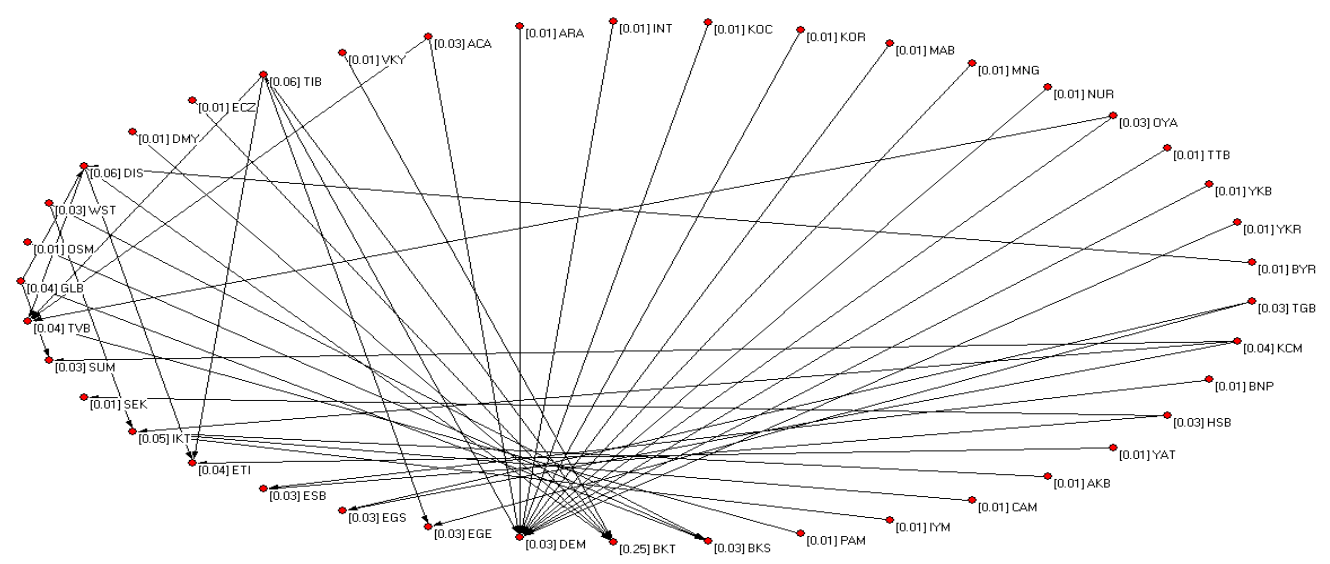

Figure 17: Circular layout representation of 1 December 2000

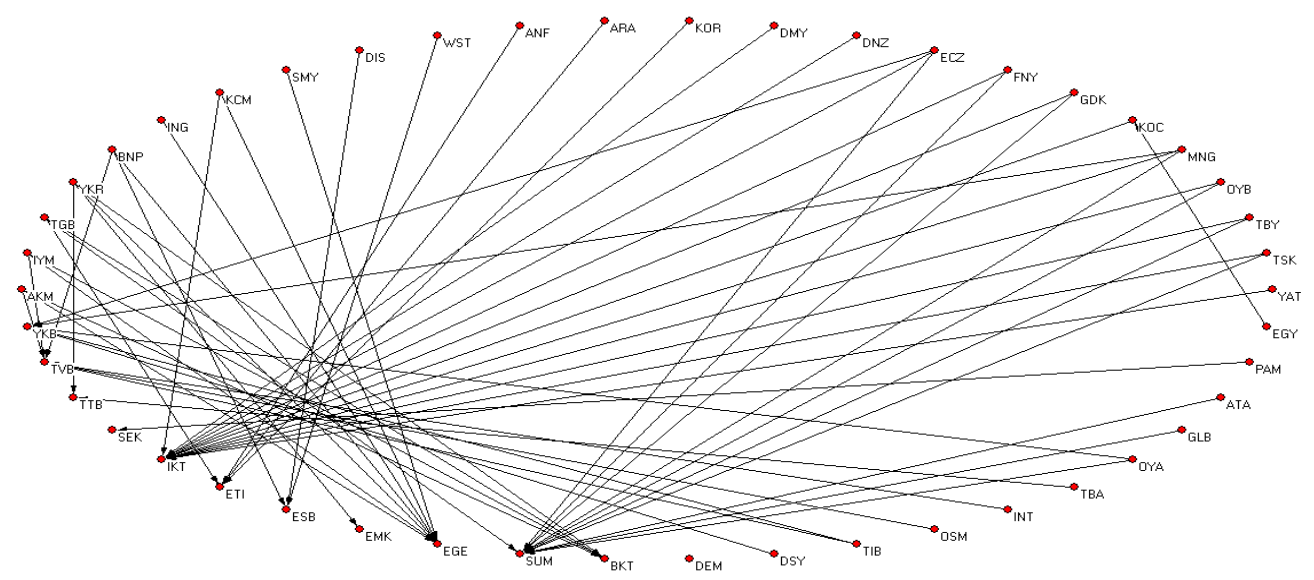

lending to Demirbank which is considered too risky.

On December 5, the takeover of Demirbank, network exhibits a multi-centered structure which represents the normalization of the interbank market. 
Figure 18: Circular layout representation of 5 December 2000

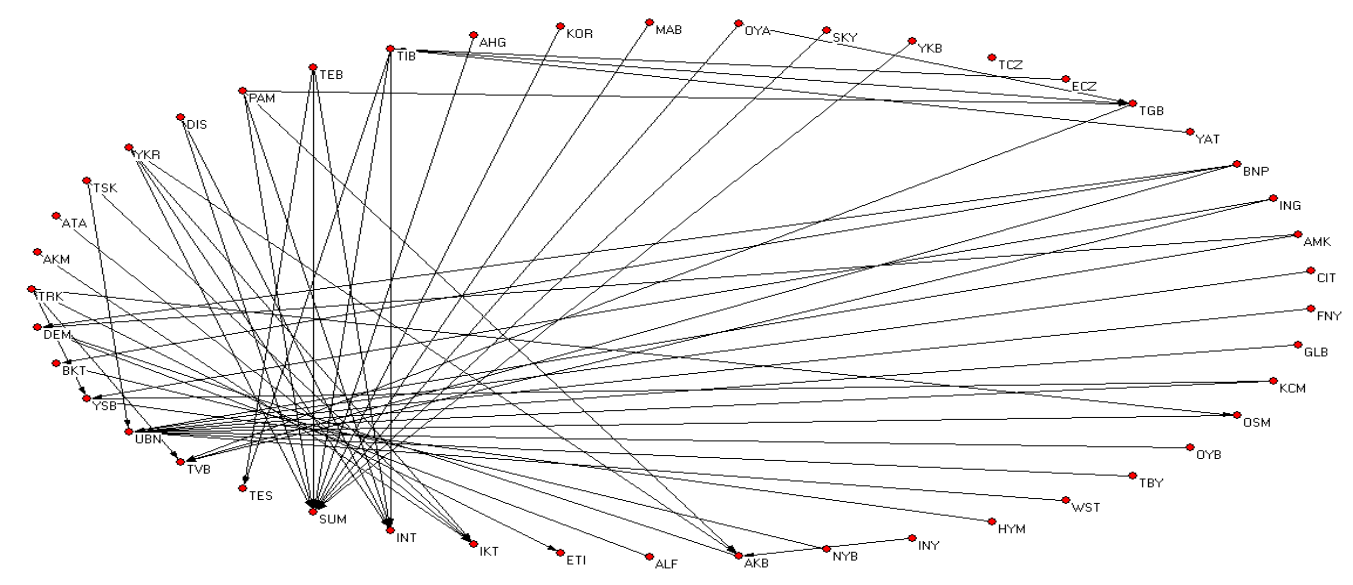

\section{Conclusion}

In this paper, we evaluate the performance of several network centrality measures in detecting systemically important financial institutions (SIFI) using data from the Turkish Interbank market during the financial crisis in 2000. We use a novel dataset from the Turkish banking crisis in 2000 which serves as a natural experiment to test network based theories of systemic risk, particularly the level of interconnections measures as centrality of an institution and its contribution to the systemic risk.

First, we provide a detailed analysis of the Turkish interbank market before and after the crisis, by relying on various network investigation statistics such as volume, transactions, links, connectivity and reciprocity. We observe a significant increase in volume and volume per transactions which is mainly due to the borrowings of Demirbank before the crisis period which drops drastically afterwards. Prior to the crisis, the total number of links in the interbank market decrease while there is an increase in the number of transactions which confirms that network becomes heavily centralized. After the crisis network evolves to a considerably less centralized one. We show that both facts in before and after crisis periods are consistently matched by connectivity and reciprocity measures. 
Exploiting the rich time dimension of our data set, we construct time series of degree, betweenness, closeness, and Bonacich centrality measures and investigate their evolution, particularly for the institution which triggered the crisis, i.e. Demirbank. We observe an increasing trend in all centrality measures for Demirbank which,as an ex-post analysis of the crisis, confirms that centrality values of institutions perform well in detecting systemically important institutions in the interbank markets. We also generate graphical illustrations of the evolution of the interbank network which shows that network is gradually becoming a star network around Demirbank before the crisis.

In this paper, due to data limitations, we only focus on the position of the institutions in the network and the weights of their links to the other institutions in the network. A more comprehensive analysis might incorporate network measures with balance sheet information which will provide us with a clearer view of defaults related to contagion and systemic risk.

\section{$7 \quad$ References}

[1] Allen, F., and D. Gale. 2000. Financial contagion. Journal of Political Economy 108 (1), $1-33$.

[2] Billio M., Getmansky M., Lo, A.W., Pellizon L. 2010 Econometric Measures of Systemic Risk in the Finance and Insurance Sectors, NBER working paper

[3] Bisias, D., Flood M., Lo A.W., Valavanis S. 2012 A Survey of Systemic Risk Analytics mimeo.

[4] Bonacich, P. 1972, Factoring and weighting approaches to clique identification, Journal of Mathematical Sociology, vol. 2, no:65, pp. 113-120. 
[5] Bravo-Benitez, B. ,Martinez Jaramillo, S., and Alexandrova-Kabadjova, B., SolorzanoMargain, J. 2012. An Empirical Study of the Mexican Banking System's Network and Its Implications for Systemic Risk Bank of Mexico Working Paper No. 2012-07

[6] Cajueiro D., and Tabak B., 2008 The role of banks in the Brazilian interbank market: Does bank type matter? Physica A 387 6825-6836

[7] Chang, E. 2008, Measures of Interbank Market Structure: An Application to Brazil, Brazilian Review of Econometrics, Vol. 28, No. 2.

[8] Cocco, J., F. Gomes, and N. Martins. 2009. Lending relationships in the interbank market. Journal of Financial Intermediation 18 (1), 24-48.

[9] Degryse, H. and G. Nguyen. 2007. Interbank exposures: An empirical examination of systemic risk in the Belgian banking system. International Journal of Central Banking 3, 951-979.

[10] Eisenberg, L., and T. Noe. 2001. Systemic risk in financial systems. Management Science 47 (2),236-249.

[11] Gai, P., and S. Kapadia. 2007. Contagion in financial networks. Working paper, Bank of England.

[12] G20 London Summit, 2009, Declaration on Strengthening the Financial System, Information Centre, http : //www.g20.org/images/stories/canalfinan/docs/uk/08deps.pdf 
[13] Gencay, R., Gradojevic, N., 2008. Overnight interest rates and aggregate market expectations, Economics Letters, 100, 27-30.

[14] Gencay, R., Selcuk, F., 2006. Overnight borrowing, interest rate risk and extreme value theory, European Economic Review, 50, 547-563.

[15] Signori, D. and Gencay, R., Economic Links and Counterparty Risk (March 14, 2012). SSRN: http://ssrn.com/abstract=2022806 or http://dx.doi.org/10.2139/ssrn.2022806

[16] J. Henggeler-Muller. 2006. The Potential for Contagion in the Swiss Interbank Market. PhD thesis, Universitat Basel,

[17] Iyer, R. and J. L. Peydro-Alcalde. 2011. Interbank Contagion at Work: Evidence from a Natural Experiment, Review of Financial Studies, 24, 1337-77, 2011.

[18] Li,S., He.J., Zhuang Y., A network model of the interbank market, Physica A, 389, 55875593

[19] Leon, C., Perez, J., 2013 Authority Centrality and Hub Centrality as metrics of systemic importance of financial market infrastructures Banco de la Repblica working papers (Central Bank of Colombia)

[20] Nier, E., Yang J., Yorulmazer T., Alentorn A,. 2007 Network models and financial stability. Journal of Economic Dynamics Control, 31 2033-2060,

[21] Saltoglu B., and Danielsson J. 2003, Anatomy of a Market Crash: A market Microstructure Analysis of the Turkish Overnight Liquidity Crisis Financial Markets Group, London School of Economics, FMG Discussion Papers 
[22] Saltoglu B, and T. Yenilmez, 2010, Analyzing Systemic Risk with Financial Networks An Application During a Financial Crash, MPRA Paper, University Library of Munich, Germany

[23] Soramaki K., Bech M., Arnold, J., Glass R., Beyeler W., 2007 The topology of interbank payment flows Physica A 379 317-333

[24] Upper, C., and A. Worms. 2004. Estimating bilateral exposures in the German interbank market: Is there a danger of contagion? European Economic Review 48 (4), 827-849.

[25] Wells, S. 2004. U.K. interbank exposures: Systemic risk implications. Journal of Monetary Economics 2 (1), 66-77. 